\title{
How we did it - an easy and feasible experimental rat model of protective role of Lipid Emulsion in Ropivacaine induced Local Anesthetic Systemic Toxicity - technique presentation and preliminary results
}

\begin{abstract}
Alexandra Lazar*, Marcel Perian, Bogdan Cordoș, Mircea Gherghinescu, Bianca Liana Grigorescu
George Emil Palade University of Medicine, Pharmacy, Science, and Technology of Targu Mures, Romania

Introduction: Local Anesthetic Systemic Toxicity (LAST) is the most feared local anesthesia accident. As the cardiac arrest determined by LAST is mostly refractory to known resuscitation protocols, due local anesthetic blockade produced in the cardiac cells, the Lipid Emulsion (L.E) has been proved to be beneficial in resuscitating the cardiac arrest determined by local anesthetic. The aim for this presentation is to ease future studies on this topic, to ensure a starting point for next related research on LAST and LE mechanism of action. Method: Under genaral anesthesia we induced Local Anesthetic Systemic Toxicity to a rat model, by injecting Ropivacaine into the inferior vena cava. We monitored the cardiac activity of the subjects during the experiment. We used 4 groups of rats, control group- no intervention, lipid group- lipid emulsion was adminsitered, local anesthetic group- local anesthetic was administered and local anesthetic and lipid emulsion group- a dose of lipid emulsion was adminsitered before administering the local anesthetic. Results: After a few attemps to incannulate teh peripheral veins we tried the more complex approach of inferior vena cava, which ensured a secure access which allowed us to repetedly adminster the local anesthetic and the lipid emulsion. Conclusion: The presented experimental animal model of induced LAST and the protective effects of LE is one of the few described in the literature, is a reproducible model, feasible, simple, low cost and can be used as starting point in future LAST research.
\end{abstract}

Keywords: animal experiment, local anesthetic, lipid emulsion, vascular access, toxicity

Received 23 November 2020 / Accepted 7 April 2021

\section{Introduction}

Regional anesthesia consists in placing local anesthetics (LA) around a nerve or in the epidural space or spinal canal. The LA acts by interrupting the pain sensation transmitted through the sensitivity fibers, which innervates a certain area of the body. All LA, except Lidocaine, are contraindicated for intravascular injection, due to their neurotoxic and cardio toxic effects (1). This is the reason why the most feared complication which can occur during a regional anesthesia, regardless of the method of administration of LA. -single shot or a continuous technique, is Local Anesthetic Systemic Toxicity (LAST). This constitutes a medical emergency because LA are known to have severe neurotoxic and cardiovascular toxicity when administered intravascular (1). Previous studies proved that when inadvertent intravascular injection occurs, LA have, firstly, neurotoxic manifestations such as convulsions, which appear at very low doses of LA. After the neurotoxic symptoms, the cardiovascular manifestations, such as arrhythmias or cardiac arrest appear. $(1,2)$. The occurrence of LAST is evaluated to be $0.03 \%$, or 0.27 episodes per 1,000 peripheral nerve blocks. (3)
The cardiac arrest determined by LAST is mostly refractory to known resuscitation protocols, due the $\mathrm{Na}$ channels blockade produced by LA in the cardiac cells. The Lipid Emulsion (LE) has been proved to be beneficial in resuscitating the cardiac arrest determined by local anesthetics. It is not yet very clear how LE works, but specific LAST resuscitation protocols, which include lipid emulsion administration, have been proposed and used worldwide (4).

The American Society of Regional Anesthesia and Pain Medicine (ASRA) created a protocol for LAST resuscitation using LE. According to this protocol, LE is recommended to be administered at the very first signs of LAST (5).

The main purpose of this paper is to create an animal based protocol of induced LAST as close as it appears in a clinical setting and an animal based protocol of LE prophylactic administration which has the potential to protect against cardiac toxic effects of LA (Ropivacaine), in case of a LAST situation.

Atso we tested the inferior vena cava approach as the venous cannulation for our drug administration, a method which is not the usual approach, due to its more complex surgical management.

Another aim for this paper is to ease future studies on this topic, to ensure a starting point for next related re- 
search on LAST induced by Ropivacaine and the LE prophylactic action against LAST.

The model we are presenting is adapted to our study, it is reproducible and adaptable to suit other studies which may be conducted on this topic.

\section{Method description}

Our study included 32 male Wistar rats, provided by the University of Medicine, Pharmacy, Science and Technology "George Emil Palade" of Tirgu-Mures, Romania Experimental Station, aged 10-12 weeks, and weighting 200-300g, randomly assigned to four groups, each group with an equal number of subjects $(n=8)$. First group was the control group, the second received 20\% LE, the third group received LA and the fourth group received both LE and LA. The subjects were acclimated to standard laboratory conditions for 14 days, respecting the circadian rhythm, and with free access to water and food intake.

All experiments were performed in accordance to established international ethical research guidelines and were approved by the Ethics Commission of the University of Medicine, Pharmacy, Science and Technology "George Emil Palade" of Tirgu-Mures, Romania (Approval No. 82/2019).

Four hours prior the anesthesia, the food and water intake were ceased. The subjects underwent general volatile anesthesia using Izoflurane (Anesteran, ROMPHARM COMPANY, Romania) in $0.5 \%-1 \%$ concentration and Oxygen-21/min. The heart rate was monitored using surface, non-invasive electrodes and the cardiac rhythm was recorded and analyzed in LabView software.

\section{Drug Dosage Calculation \\ Lipid Emulsion}

For Lipid Emulsion dose calculation, we used the protocol from ASRA adapted for our subjects, due to the inexistence of standardized protocols of lipid administration and dosage in rats. We converted the recommended dose for an approximatively $70 \mathrm{~kg}$ person, to each subject, related to their weight, as following:

$70 \mathrm{~kg}=70000 \mathrm{~g} \ldots \ldots \ldots \ldots \ldots \ldots \ldots . \ldots 100 \mathrm{ml} / \mathrm{lipid} 20 \%$

Rat's weight g......................... ml/ lipid 20\%

$$
\mathrm{X}=\frac{\text { rat }^{\prime} \text { s weight } \mathrm{x} 100 \mathrm{ml}}{70000 \mathrm{~g}}
$$

For our study groups the lipid dose ranged between 0.3$0.4 \mathrm{ml}$, according to their weight, for each subject the dose was calculated according to the equation presented above. The dose of the LE was administered as a bolus, because in clinical settings this drug is not recommended to be continuously perfused, just for prophylactic purposes, due to its possible side effects (6). The aim of our study is to evaluate the prophylactic use of this drug in case of LAST, on an animal based model.

\section{Local Anesthetic}

The dosage of LA which will determine cardiac toxic effects it is not precisely established, various dosages being able to produce cardiac toxic effects. We titrated the LA dose to obtain side effects such as arrhythmias, without producing cardiac arrest. After trying multiple dosages, we concluded that a dose of $0.2 \mathrm{mg}$ Ropivacaine $(0.1 \mathrm{ml}$ of $20 \%$ solution $)$ administered intravenously, generates toxic cardiac effects in rats, such as heart blocks, ventricular fibrillation, without producing cardiac arrest in the subjects. We chose to administer boluses of LA because this is the the most common mean by which LAST is induced in clinical setting; the LA enters the systemic circulation by accident, continuous intravenous Ropivacaine perfusions are contraindicated in humans (7).

\section{Method}

Before starting the experiment, all the subjects were anesthetized in the anesthesia chamber, then they were transferred on the surgery table when the anesthesia was complete (Figure 1).

The cardiac activity was monitored using noninvasive surface electrodes, for all the subjects. The inferior vena cava was cannulated after exposing it through a median laparotomy. After inferior vena cava identification, a 26 $G$ catheter was inserted and maintained in place until the experiment was finished (Figure 1). Normal fluid balance was maintained by infusion of $0.9 \%$ saline according to the international recommendation (8).

The subjects in the control group received only normal saline $(2 \mathrm{ml})$.

The subjects in group 2 received a bolus of 0.3 or $0.4 \mathrm{ml}$ of lipid solution 20\% (Fresenius Kabi, Romania), according to the presented equation.

The subjects in group 3 received local anesthetic - Ropivacaine (Fresenius Kabi, Romania) 200mg/20 ml - 2mg Ropivacaine/ml, in $0.1 \mathrm{ml}$ boluses; up to a total of 4 boluses were administered, at 2 minutes intervals and the appearance of arrhythmias was recorded (Figure 2).

For the subjects in group 4 we administered the calculated lipid emulsion dosage and administered it as a bolus, before LA, trough the $26 \mathrm{G}$ inserted catheter. After 2 minutes we administered 4 cycles of $0.1 \mathrm{ml}$ Ropivacaine at 120 seconds intervals. If the subject developed asystole during the experiment, the protocol was discontinued.

\section{Results}

\section{Venous cannulation technique}

The technique of cannulating the inferior vena cava, although needs more practical skills, could be of choice, since this approach proved to be safer than other peripheral venous cannulation approaches - tail or paw venous cannulation. It allowed us to have a stable venous access, the 26 cannula could be secured and allowed multiple administrations even in critical conditions, such as severe arrhythmias, when the systemic circulation was unstable. 


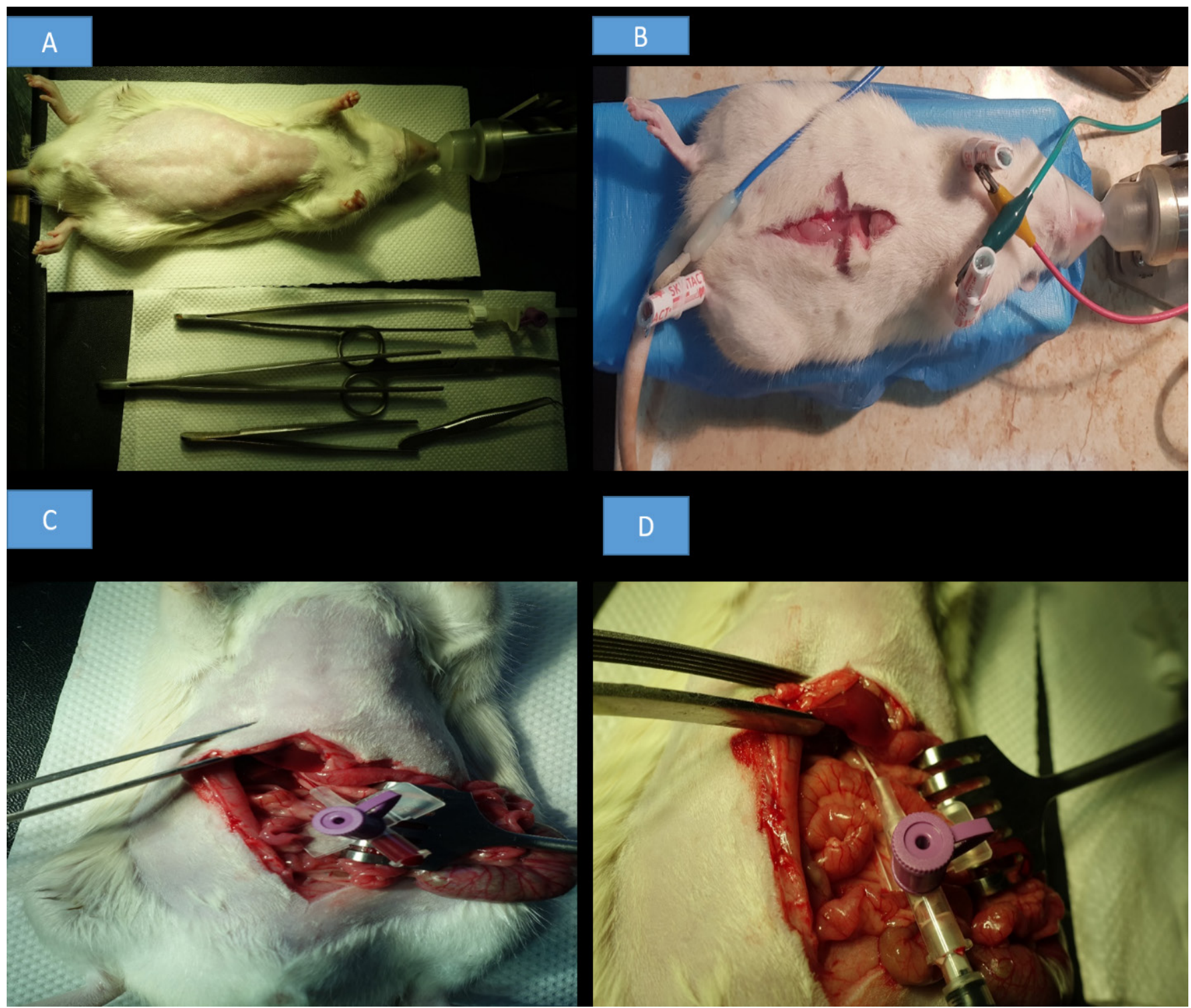

Fig. 1. Surgical procedure for venous cannulation and lipid administration: A- procedure preparation; B-incision site and cardiac monitoring of the subjects; C- inferior vena cava cannulation; D- Lipid Emulsion administration.

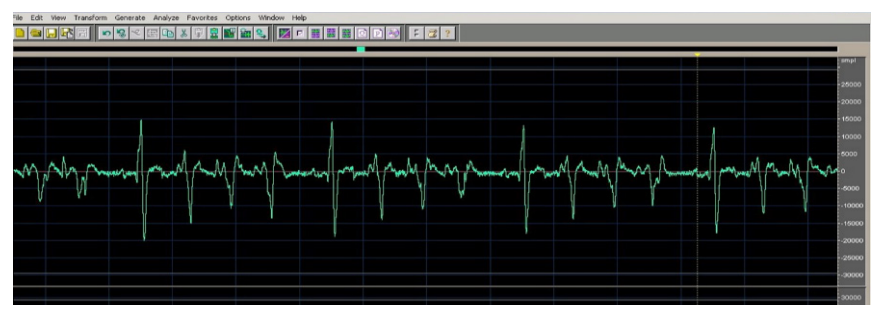

Fig. 2. Cardiac effects determined by intravascular LA administration.

\section{Lipid Emulsion prophylactic administration}

When administered alone, without being followed by LA, the LE did not affect in any way the cardiac function of the subjects. The rate and the rhythm remained close to the basal values.

When administered and followed by LA, the LE showed some protective effects, the subjects in group 4, although presented cardiac arrhythmias, they did not lead to cardiac arrest as frequent as in group 3, where the LE was not administered.

\section{Discussion}

Local anesthetic systemic toxicity is a life-threatening emergency. Fortunately, it is not that often encountered, though when appears it can lead to patient's death (9). Experimental studies on humans on this medical emergency is very difficult to conduct, yet there were a few studies of induced LAST to humans, in healthy volunteers $(10,11)$. But these studies were not on large number of patients and as it is known human variability is a very important factor in obtaining clear results. This is where animal experimental models become very useful, because the animals used in experimental medicine are assumed to be genetically identical.

To be able to simulate LAST, as close as to a clinical situation, we needed to inject the LA into systemic circulation. Most of the experimental studies on this matter, used larger animals due to the easiest venous access and monitoring (12). This type of experiments is very expensive, and we tried a feasible and more accessible model of animal 
study using smaller subjects (rats). The translation from a bigger animal model to a smaller one was difficult due to the fragile and poor represented peripheral vascular system.

At the beginning of our quest for vascular access, we first tried the caudal vein, as well as the veins from the paws. Neither of these were satisfactory to our needs because we could not maintain the access for longer periods of time, even when using peripheral catheters of 26G. We needed to be able to inject multiple times, because we had to administer several boluses of LE and LA, therefore we needed a stable venous access in place.

The solution we came up was the use of the vena cava inferior. This approach is more technical and complex than the peripheral one, but it ensured us a safe vascular approach and provided a permanent access needed for the completion of the experiment.

Since the dosage of LA which will determine cardiac toxic effects it is not precisely established, various dosages being able to produce cardiac toxic effects, we titrated the LA dose with the intention of obtaining side effects such as arrhythmias, without producing cardiac arrest. After trying multiple dosages, we concluded that a dosage of $0.1 \mathrm{ml}$ of Ropivacaine, which is $0.2 \mathrm{mg}$ of LA administered intravenous, produces toxic cardiac effects in rats without cardiac arrest.

Although the study needs to be continued, this being a preliminary report, LE showed some protective effects on LA induced LAST, when administered before LA, aspect which can be taken into consideration and has the potential of being implemented in clinical practice, as a prophylactic method.

The obstacle which needed to be overcome in these studies, on LAST, is that the experiment should be as close as possible to what a human body may experience in the given situation. This model has the advantage of being cheap, smaller animals being cheaper than the large ones and also the cost of housing them is lower. The disadvantage can be that there is the need qualified personnel to work with them, as with any other animal model and also that some inter-specie variability may occur.

The toxic effects of LAST are life threatening this being the reason why studies on human subjects are dangerous, due to cardiotoxic and neurotoxic effects (1). Creating a simple feasible and low cost animal model for LAST and the protective effects of LE is an important step forward towards a more profound understanding of the mechanisms involved in LAST appearance and eases the path for newer approaches in protective management this syndrome.

A limitation of this experiments consists of the lack of neurologic monitoring technology to observe early neurological signs induced by LAST, which are known to appear first (2). This may be a serious obstacle to overcome because the experiment, due to ethical issues, should produce minimum stress to the animal, and this desiderate can be achieved in general anesthesia, which will interfere with any neurological signs.
Our protocol for animal induced LAST, in this approach of creating LAST similar to clinical setting, it is, to our knowledge, one of the very few on this matter. It can be updated, and it can be modified according to other researchers needs, because the matter of safety in anesthesia, and in this particular care, regional anesthesia, is of high interest nowadays. We tried to induce the syndrome as close as possible and observe the cardiovascular modification determined by LA as well the protective effects of LE administration.

\section{Conclusion}

The presented experimental animal model of induced LAST and the protective effects of LE is a reproducible model, feasible, simple, low cost and can be used as starting point in future LAST research.

The use of inferior vena cava approach, as venous access, although more technical, ensures a safe and stable vascular access throughout the entire procedure.

\section{Aknowledgement}

This work was supported by the University of Medicine, Pharmacy, Science and Technology of Târgu Mureș Research Grant number 615/9/17.01.2019.

\section{Authors contribution:}

A.L- substantial contributions to conception and design, acquisition of data analysis and interpretation of data, drafting the article, final approval of the version to be published

P.M- substantial contributions to conception and design, acquisition of data substantial contributions to acquisition of data, drafting the article, final approval of the version to be published

C.B- substantial contributions to acquisition of data, drafting the article, final approval of the version to be published M.G- substantial contributions to acquisition of data, drafting the article, final approval of the version to be published

B.L.G- substantial contributions to conception and design, substantial contributions to revising the article critically for important intellectual content, final approval of the version to be published

\section{References}

1. Lirk P, Picardi S, Hollmann MW. Local anaesthetics: 10 essentials Eur J Anaesthesiol. 2014; 31(11):575-85.

2. Yang S, Abrahams MS, Hurn PD, Grafe MR, Kirsch JR. Local anesthetic Schwann cell toxicity is time and concentration dependent. Reg Anesth Pain Med. 2011; 36(5):444-51.

3. El-Boghdadly K, Pawa A, Chin KJ. Local anesthetic systemic toxicity: current perspectives. Local Reg Anesth. 2018;11:35-44.

4. Ok SH, Hong JM, Lee SH, Sohn JT. Lipid Emulsion for Treating Local Anesthetic Systemic Toxicity. Int J Med Sci. 2018;15(7):713-722.

5. Rubin DS, Matsumoto MM, Weinberg G, et al Local Anesthetic Systemic Toxicity in Total Joint Arthroplasty: Incidence and Risk Factors in the United States From the National Inpatient Sample 1998-2013 Regional Anesthesia \& Pain Medicine 2018;43:131-137.

6. https://www.accessdata.fda.gov/drugsatfda_docs/label/2007/017643 s072,018449s039lbl.pdf 
7. https://www.accessdata.fda.gov/drugsatfda_docs/label/2012/ 020533s028lbl.pdf

8. Karl-Heinz Diehl, Robin Hull, David Morton et al. A Good Practice Guide to the Administration of Substances and Removal of Blood, Including Routes and Volumes. J. Appl. Toxicol.2001;21:15-23

9. Verlinde M, Hollmann MW, Stevens MF, Hermanns $H$, Werdehausen R, Lirk P. Local Anesthetic-Induced Neurotoxicity. Int J Mol Sci. 2016;17(3):339.
10. https://www.asra.com/advisory-guidelines/article/3/checklist-fortreatment-of-local-anesthetic-systemic-toxicity

11. McCartney CJ, Murphy DB, lagounova A, Chan WW. Intravenous ropivacaine bolus is a reliable marker of intravascular injection in premedicated healthy volunteers. Can J Anaesth. 2003;50(8):795-800.

12. Kessler J, Marhofer P, Hopkins PM, Hollmann MW. Peripheral regiona anaesthesia and outcome: lessons learned from the last 10 years. $\mathrm{Br} J$ Anaesth. 2015; 114(5):728-45. 